\title{
Correction to: Runtime self-monitoring approach of business process compliance in cloud environments
}

\author{
Ahmed Barnawi $^{1} \cdot$ Ahmed Awad $^{2} \cdot$ Amal Elgammal $^{2} \cdot$ Radwa El Shawi $^{3} \cdot$ Abdullah Almalaise $^{1} \cdot$ Sherif Sakr $^{4}$
}

Published online: 28 February 2018

(c) Springer Science+Business Media, LLC, part of Springer Nature 2018

\section{Correction to: Cluster Comput (2015) 18:1503-1526 \\ https://doi.org/10.1007/s10586-015-0494-0}

The original version of this article unfortunately contained a mistake in the acknowledgement statement. It should read as follow "This project was funded by the National Plan for
Science, Technology and Innovation (MAARIFAH) - King Abdulaziz City for Science and Technology - the Kingdom of Saudi Arabia - award number (11-INF1991-03). The authors also, acknowledge with thanks Science and Technology Unit, King Abdulaziz University for technical support".

The original article can be found online at https://doi.org/10.1007/ s10586-015-0494-0.

$\triangle$ Ahmed Awad a.gaafar@fci-cu.edu.eg

Ahmed Barnawi

ambarnawi@kau.edu.sa

Amal Elgammal

a.elgammal@fci-cu.edu.eg

Radwa El Shawi

rmelshawi@pnu.edu.sa

Abdullah Almalaise

aalmalaise@kau.edu.sa

Sherif Sakr

sakrs@ksau-hs.edu.au

1 King Abdulaziz University, Jeddah, Saudi Arabia

2 Cairo University, Giza, Egypt

3 Princess Nourah Bint Abdulrahman University, Riyadh, Saudi Arabia

4 King Saud bin Abdulaziz University for Health Sciences, Riyadh, Saudi Arabia 